\title{
Psicooncología
}

ISSN: 1696-7240

\section{Desarrollo de la escala EDSOL para la detección de la soledad existencial en enfermos al final de la vida}

\author{
Silvia Viel Sirito ${ }^{1}$; Jorge Maté Méndez ${ }^{2,8}$; Dolors Mateo Ortega ${ }^{3,8}$; Montserrat Bernaus Martí ${ }^{4}$; Cruz \\ Sánchez Julvé5; Jaume Martínez-Romans; ; María José Gómez-Romero7; Joaquín T. Limonero ${ }^{8}$
}

Recibido: 29 de septiembre de 2017 / Aceptado: 23 de enero de 2018

Resumen. Objetivo: Presentar el desarrollo de la escala de detección de la soledad existencial (EDSOL) en personas con enfermedades avanzadas en tratamiento paliativo. Método: Se describe el proceso de elaboración de la escala Detección de la Soledad Existencial EDSOL. Para ello, 1) se ha revisado la bibliografía existente sobre el tema; 2) se ha establecido un marco teórico de referencia; 3) se han definido los criterios que debía cumplir el instrumento de cribado; y 4) se ha consultado con expertos y enfermos la adecuación de los ítems de la escala. Resultados: Se presenta a la escala EDSOL, que consta de dos partes: 1) tres preguntas dirigidas a los enfermos; y 2) diferentes observaciones realizadas por el personal sanitario acerca de la presencia de signos externos de soledad existencial. La escala final fue revisada por enfermos y profesionales. Conclusión: La escala EDSOL es una herramienta apropiada para identificar la presencia de Soledad Existencial en pacientes al final de la vida. En este caso, se propondrá su uso sistemático en la detección temprana de la Soledad Existencial y la implementación de intervenciones asistenciales específicas.

1 Silvia Viel Sirito. Grupo de Psicólogos Sociedad Catalana-Balear de Cuidados Paliativos. Grupo de Investigación en Estrés y Salud (GIES). Facultad de Psicología. Universidad Autónoma de Barcelona, (Bellaterra, Barcelona). E-mail: Silviaviel@hotmail.com

2 Jorge Maté Méndez. Institut Català d'Oncologia. Unidad de Psicooncología (L'Hospitalet de Llobregat, Barcelona). Grupo de Investigación en Estrés y Salud (GIES). Facultad de Psicología. Universidad Autónoma de Barcelona, (Bellaterra, Barcelona).

E-mail: jmate@iconcologia.net

3 Dolors Mateo Ortega. Consorci Sanitari de Terrassa. Unidad de Cuidados Paliativos. Unidad de Geriatría (Terrassa, Barcelona). Grupo de Investigación en Estrés y Salud (GIES). Facultad de Psicología. Universidad Autónoma de Barcelona, (Bellaterra, Barcelona).

E-mail: DMateo@CST.CAT

4 Montserrat Bernaus Martí. Corporació Sanitària Parc Taulí (Sabadell, Barcelona). Unidad de Cuidados Paliativos (Sabadell, Barcelona).

E-mail: MBernaus@tauli.cat

5 Cruz Sánchez. Consorci Sanitari del Garraf, Hospital-Residència Sant Camil (Sant Pere de Ribes, Barcelona). E-mail: Csanchezj@csg.cat

6 Jaume Martínez-Romans. Servicio de Oncología. Unidad de Psicooncología. Hospital de la Vall d'Hebron. (Barcelona). E-mail: Jaumarti@vhebron.net

7 María José Gómez-Romero. Unidad de Psicología. Egarsat. Mutua Colaboradora con la Seguridad Social $n^{\circ}$ 276. (Terrassa. Barcelona). Grupo de Investigación en Estrés y Salud (GIES). Facultad de Psicología. Universidad Autónoma de Barcelona, (Bellaterra, Barcelona).

E-mail: mjgomez@egarsat.es

8 Joaquín T. Limonero. Grupo de Investigación en Estrés y Salud (GIES). Facultad de Psicología. Universidad Autónoma de Barcelona, (Bellaterra, Barcelona).

E-mail: Joaquin.limonero@uab.cat

* Dirección de correspondencia: Dr. Jorge Maté-Méndez. Institut Català d'Oncologia. Unidad de Psicooncología. Granvia de L'Hospitalet, 199-203, 08908 L'Hospitalet de Llobregat, Barcelona, España.

Email: jmate@iconcologia.net 
Palabras clave: Enfermo al final de la vida; sufrimiento existencial; soledad existencial; escala de evaluación; cuidados paliativos.

\title{
[en] Development of the EDSOL scale for the detection of existential loneliness in patients at end of life
}

\begin{abstract}
Aim: This paper presents the development of Detection of Existential Loneliness Scale (EDSOL) of patients with advanced disease/terminal illness that receiving palliative care. Method: The process of preparing the scale is described. For this: 1) the literature on the subject has been revised, 2) theoretical framework has been established, 3 ) it has been defined the criteria to be met by the screening tool, and 4) the final scale was reviewed by patients and experts. Results: The EDSOL scale consists of two parts: 1) three questions addressed to patients and; 2) several questions addressed to health staff about the observation of external signs of Existential Loneliness. The final scale was reviewed by patients and experts (facie validity) showing good validity. Conclussion: The EDSOL scale will be an appropriate tool for identifying the Existential Loneliness of patients at the end of life or advanced illness. In this case, we will propose the systematic use of EDSOL for early detection and specific interventions on existential loneliness experiences.
\end{abstract}

Keywords: Patient at the end of life; existential suffering; existential loneliness; assessment scale; palliative care.

Sumario. 1. Introducción 2. Material y métodos 3. Resultados a) Descripción de la escala (EDSOL) 4. Discusión 5. Financiación 6. Conflicto de intereses. 7. Referencias bibliográficas.

Cómo citar: Viel Sirito S, Maté Méndez J, Mateo Ortega D, Bernaus Martí M, Sánchez Julvé C, Martínez-Romans J, Gómez-Romero MJ, Limonero JT. Desarrollo de la escala EDSOL para la detección de la soledad existencial en enfermos al final de la vida. Psicooncología 2018;15:89-101. Doi: 10.5209/ PSIC.59178.

\section{Introducción}

La Organización Mundial de la Salud ${ }^{(1)}$ estima que alrededor del $80 \%$ de 1 os 7,5 millones de personas que mueren cada año de cáncer serían susceptibles de beneficiarse de cuidados paliativos, pudiendo prevenir y aliviar su sufrimiento al final de la vida favoreciendo que el enfermo muera con la mayor dignidad posible y ayudar a sus allegados tras la pérdida. En este sentido, la enfermedad en fase avanzada/terminal se define como aquella enfermedad incurable, avanzada, progresiva con pronóstico de vida limitado, escasa posibilidad de respuesta a tratamientos específicos, que presenta una evolución de carácter oscilante y frecuentes crisis de necesidades, y que provoca un intenso impacto emocional y familiar con repercusiones sobre la estructura cuidadora que condicionan una alta demanda y uso de recursos ${ }^{(2)}$. Estas enfermedades que amenazan la vida, como es el caso del cáncer se caracterizan por una serie de síntomas y experiencias que pueden generar sufrimiento en los enfermos y en sus cuidadores ${ }^{(3)}$. Para poder aliviar el sufrimiento de estas personas se desarrollaron los cuidados paliativos cuyos principios fundamentales consisten en la atención integral de los enfermos y de su familia, a través de una asistencia global que abarca las necesidades biomédicas, psicológicas, sociales y espirituales/ existenciales del enfermo, familia y cuidadores ${ }^{(4)}$. Es decir, su fin último es el alivio del sufrimiento y la mejoría del bienestar. 
Diferentes autores se han referido a la experiencia de sufrimiento al final de la vida. Entre ellos Cassell (5), que afirma "Los que sufren no son los cuerpos, son las personas", acentuando la dimensión personal, subjetiva, del sufrimiento al final de la vida. Por su parte, Chapman y Gavrin ${ }^{(6)}$ indican que el sufrimiento es la amenaza percibida a la integridad de uno mismo (self) junto con la percepción de desamparo frente a esa amenaza.

Una definición más integradora es la que realizan Bayés y colaboradores ${ }^{(7)}$, en donde el sufrimiento que experimenta el paciente al final de la vida es una experiencia absolutamente individual, subjetiva y dependerá del grado de amenaza que representen los problemas o situaciones vividas durante el transcurso de la etapa final de su vida. En este sentido, Krikorian, Limonero, Román, Vargas y Palacio ${ }^{(8)}$ señalan que es una experiencia multidimensional y dinámica de estrés severo que se produce ante la percepción de amenaza a la integridad de la persona y en donde los procesos regulatorios (psicológicos y conductuales), que normalmente llevarían a la adaptación, resultan insuficientes y causan agotamiento ${ }^{(8,9)}$.

A partir del análisis de las diferentes aproximaciones conceptuales sobre el sufrimiento, se desprende que las causas del mismo pueden ser múltiples, diversas, dinámicas, sincrónicas y tener efectos aditivos ${ }^{(3,8-10)}$.

La experiencia de una enfermedad avanzada invade a toda la persona que la padece, afectando el área física, psicológica, social, espiritual y/o existencial. Así, se ha relacionado dolor espiritual o existencial ${ }^{(11,12)}$. Hacer frente a una enfermedad, sin esperanza de curación o a una situación de final de vida, puede situar a la persona en una posición existencial de ruptura con sus vínculos emocionales y valores personales ${ }^{(11)}$.

La soledad existencial se podría definir como un sentimiento de soledad que se experimenta cuando una persona se siente sola a pesar estar acompañado por otras personas $^{(13)}$. No obstante, una definición más completa incluiría ${ }^{(14-16)}$ : a) soledad social, caracterizada por el sentimiento de tristeza y pertenencia que resulta de una carencia de redes sociales; a menudo se presenta ante situaciones de pérdida de la salud, disminución de redes sociales, pérdida de roles sociales, pérdida de pareja, duelos familiares, etc.; b) soledad emocional, que hace referencia al sentimiento relacionado con la experiencia de sentirse solo aún ante la presencia de otros; este sentimiento se relaciona con la pérdida de una figura de apego. Las personas al final de la vida, pueden tener dificultades en comprender y expresar las emociones relacionadas con la proximidad de la muerte; y c) soledad existencial, que hace alusión al sentimiento asociado a la experiencia de sentirse vacío, triste, sin contar con un vínculo, que resulta de la toma de conciencia de existir separados de los demás.

Algunos autores para comprender mejor la soledad existencial distinguen tres dimensiones: condición, experiencia emocional siempre frente la muerte; experiencia -mecanismo de defensa, sentimiento o toma de conciencia, difícil de comunicar-y proceso-que incluiría crecimiento interno, encontrar sentido ${ }^{(17)}$ - mientras que para otros la representan en tres categorías: interpersonal (social), intrapersonal y existencial ${ }^{(18,19)}$. En una línea de complejidad creciente Yalom ${ }^{(20)}$ englobaría la soledad existencial en un concepto más amplio y holístico como sería el sufrimiento existencial.

Diversos autores señalan la presencia de la soledad existencial como uno de los factores que puede transformar el proceso de morir en una experiencia dolorosa que puede producir altos niveles de malestar emocional ${ }^{(12,19)}$, en especial cuando no pueden hablar abiertamente sobre sus pensamientos o preocupaciones sobre la vida y la muerte ${ }^{(13)}$. 
En la medida que la soledad existencial es generadora de malestar emocional, su identificación temprana resulta primordial para la atención especializada, sobre todo de enfermos al final de la vida ${ }^{(18,19)}$. Así por ejemplo, se observa que la posibilidad de mantener vínculos significativos con otros en este trance vital es un factor crucial para poder morir en paz $^{(21,22)}$.

Teniendo en cuenta: 1) la necesidad de evaluación de la soledad; y 2) la carencia de instrumentos adecuados de evaluación para medirla que se adapten tanto a las necesidades de la práctica clínica diaria como al entorno de los cuidados paliativos y de los enfermos al final de la vida, el objetivo principal del presente trabajo consiste en la elaboración de un instrumento para la detección de la soledad existencial en pacientes con enfermedad avanzada al final de vida. De acuerdo con el objetivo principal, 1) se describe el proceso sistemático de elaboración de la Escala de Detección de la Soledad Existencial (EDSOL) de enfermos con enfermedad avanzada al final de la vida atendidos en una Unidad de Cuidados Paliativos (UCP); y 2) se presenta la escala EDSOL en su versión final.

\section{Material y métodos}

A continuación se describen los pasos realizados de manera cronológica para el desarrollo de la escala EDSOL:

1. Formación de un equipo de trabajo. Ocho psicólogos con experiencia en cuidados paliativos pertenecientes al Grupo de Psicólogos de la Sociedad Catalano-Balear de Cuidados Paliativos formaron parte de un grupo de trabajo específico interesado en el estudio de la soledad existencial.

2. Revisión de la literatura. Se revisó la bibliografía relevante sobre el tema en frecuentes reuniones presenciales y seguimiento online. Se consultaron las bases de datos electrónicas Medline, Scopus y Crochane, así como documentos oficiales como guías, manuales o tesis doctorales publicados o inéditas comprendidas entre el año 1990 y el 2016. Las palabras claves que se utilizaron fueron: loneliness, existential loneliness, existential suffering, psychological distress, emocional distress, subjective assessment, psychological assessment, emotional assessment, palliative care, palliative care, palliative medicine y sus consiguientes homólogos en castellano (soledad, soledad existencial, sufrimiento existencial, malestar emocional, distrés emocional, evaluación subjetiva, evaluación psicológica, evaluación emocional, cuidados paliativos, cuidados paliativos, medicina paliativa).

3. Establecimiento de los criterios que debía cumplir la escala EDSOL de acuerdo con las propuestas de otros autores para la elaboración de escalas en el ámbito de los cuidados paliativos ${ }^{(23-27)}$ : a) ser sencillo y de fácil aplicación; b) adaptado al lenguaje del enfermo; c) la administración debe ser capaz de generar efectos terapéuticos y nunca iatrogénicos; d) que se evalúen aspectos relevantes; e) que contengan pocas preguntas; f) que el tiempo de aplicación sea breve; g) que monitoricen la evolución del sentimiento de soledad existencial en el tiempo; y h) que faciliten su derivación, si fuera necesario, a un profesional especializado para una evaluación e intervención específicas y además poseer buenas propiedades psicométricas ${ }^{(23)}$. 
4. Establecimiento de un marco teórico de referencia. En la elaboración de la EDSOL se partió del modelo Integrativo del Sufrimiento ${ }^{(28)}$ que tiene su origen en modelos precedentes exitosos como el modelo Amenazas-Recursos ${ }^{(7)}$ así $^{2}$ como en las escalas de cribado que nuestro grupo ha ido desarrollando ${ }^{(24-26)}$. Se ha profundizado en los factores determinantes del sufrimiento existencial, con especial énfasis a la Soledad Existencial presente en enfermos/familias al final de la vida.

5. Establecimiento de la validación de contenido. Esta validación se realizó por profesionales y por enfermos. En el primer caso, el diseño preliminar de la escala se sometió a un grupo de profesionales expertos en $\mathrm{CP}$ que valoraron la idoneidad de las preguntas y si éstas debían ser modificadas o reformuladas. Para la validación con enfermos, la evaluación de concordancia entre los jueces se realizó mediante la validez de facie o aparente. Se presentó la escala a un grupo reducido de enfermos y se les preguntó si las preguntas eran comprensibles, si las consideraba importantes, si faltaba algún aspecto relevante que no se hubiera preguntado, y si se consideraba alguna inadecuada o les había molestado las preguntas realizadas.

\section{Resultados}

La escala EDSOL se ha elaborado teniendo en cuenta la revisión bibliográfica, la discusión de diferentes modelos teóricos, la búsqueda de consenso entre los miembros del Grupo de Trabajo y la validación de contenido de profesionales y de enfermos.

De la revisión bibliográfica centrada en instrumentos elaborados para la detección del sufrimiento existencial al final de la vida, observamos que la mayoría de ellos no cumplían con nuestros criterios. En gran número destacaban por contener o un número excesivo de ítems y/o no eran específicos para evaluar estos aspectos en enfermos al final de la vida.

En base a la inexistencia de instrumentos adecuados, y específicos para la evaluación de la soledad existencial, se elaboró una escala específica para la evaluación la misma en pacientes con enfermedad avanzada al final de vida (EDSOL) (Ver Figura 1).

\section{a) Descripción de la escala (EDSOL)}

La escala elaborada para evaluar la soledad existencial (EDSOL) consta de 2 partes: una dirigida al enfermo, y otra dirigida al profesional sanitario. Con relación al enfermo, se evalúa la percepción del sentimiento de soledad a través de 3 preguntas con formato de respuesta tipo likert de 6 puntos y una pregunta abierta en cada una de ellas preguntando el por qué de la respuesta emitida. La segunda parte consiste en diferentes observaciones por parte del profesional sanitario acerca de la presencia de signos externos de soledad, como pueden ser comportamientos relacionados con la demanda o rechazo de visitas, dificultad para separarse de los seres queridos, signos visibles de llanto, miedo, tristeza o de enfado, entre otros. Se cuantifican estos signos externos en una escala de intensidad de 0 a 5. 
Fecha de administración:

Hora de administración:

Código / Número:

Evaluador:

DETECCIÓN DE SOLEDAD EXISTENCIAL (EDSOL), página 1 de 2

1a. ¿Últimamente se ha sentido solo?

\begin{tabular}{|c|c|c|c|c|c|}
\hline NUNCA & $\begin{array}{c}\text { CASI } \\
\text { NUNCA }\end{array}$ & $\begin{array}{c}\text { ALGUNAS } \\
\text { VECES }\end{array}$ & $\begin{array}{c}\text { MUCHAS } \\
\text { VECES }\end{array}$ & $\begin{array}{c}\text { CASI } \\
\text { SIEMPRE }\end{array}$ & SIEMPRE \\
\hline$\square$ & $\square$ & $\square$ & $\square$ & $\square$ & $\square$ \\
\hline
\end{tabular}

1b. Por qué? (independiente de las respuestas que dé el paciente se le pregunta el porqué).

2a. ¿En qué medida se ha sentido solo?

\begin{tabular}{|c|c|c|c|c|c|}
\hline NADA & UN POCO & $\begin{array}{c}\text { NI MUCHO } \\
\text { NI POCO }\end{array}$ & BASTANTE & MUCHO & MUCHÍSIMO \\
\hline$\square$ & $\square$ & $\square$ & $\square$ & $\square$ & $\square$ \\
\hline
\end{tabular}

2b. Por qué? (independiente de las respuestas que dé el paciente se le pregunta el porqué).

3a. En general, ¿disfruta de la compañía, contacto o relación con los demás?

\begin{tabular}{|c|c|c|c|c|c|}
\hline NUNCA & $\begin{array}{c}\text { CASI } \\
\text { NUNCA }\end{array}$ & $\begin{array}{c}\text { ALGUNAS } \\
\text { VECES }\end{array}$ & $\begin{array}{c}\text { MUCHAS } \\
\text { VECES }\end{array}$ & $\begin{array}{c}\text { CASI } \\
\text { SIEMPRE }\end{array}$ & SIEMPRE \\
\hline$\square$ & $\square$ & $\square$ & $\square$ & $\square$ & $\square$ \\
\hline
\end{tabular}

3b. Por qué? (independiente de las respuestas que dé el paciente se le pregunta el porqué). 
Fecha de administración:

Hora de administración:

Código / Número:

Evaluador:

DETECCIÓN DE SOLEDAD EXISTENCIAL (EDSOL), página 2 de 2

4a. ¿Con qué frecuencia el enfermo se encuentra acompañado?

\begin{tabular}{|c|c|c|c|c|c|}
\hline NUNCA & $\begin{array}{c}\text { CASI } \\
\text { NUNCA }\end{array}$ & $\begin{array}{c}\text { ALGUNAS } \\
\text { VECES }\end{array}$ & $\begin{array}{c}\text { MUCHAS } \\
\text { VECES }\end{array}$ & $\begin{array}{c}\text { CASI } \\
\text { SIEMPRE }\end{array}$ & SIEMPRE \\
\hline$\square$ & $\square$ & $\square$ & $\square$ & $\square$ & $\square$ \\
\hline
\end{tabular}

4b. Las personas que acompañan al enfermo son:

\begin{tabular}{|c|c|c|c|c|c|}
\hline \multicolumn{5}{|l|}{ SIEMPRE LA MISMA PERSONA } \\
\hline PAREJA & HIJO & HIJA & HERMANO/A & NIETO/A & OTRA \\
\hline$\square$ & $\square$ & $\square$ & $\square$ & $\square$ & \\
\hline
\end{tabular}

\begin{tabular}{|c|c|c|c|c|c|c|c|}
\hline \multicolumn{2}{|c|}{ PERSONAS DIFERENTES } & \multicolumn{3}{|c|}{$\square$ POCAS } & \multicolumn{3}{c|}{ MUCHAS } \\
\hline PAREJA & HIJO & HIJA & HERMANO/A & NIETO/A & OTRO FAM & AMIGO & OTRA \\
\hline$\square$ & $\square$ & $\square$ & $\square$ & $\square$ & $\square$ & $\square$ & \\
\hline
\end{tabular}

\section{SIGNOS EXTERNOS DE SOLEDAD EXISTENCIAL}

a. ¿Consideras que el enfermo presenta signos externos de soledad?

p. ej. comportamientos, verbalizaciones, etc. $\square$ Sí $\square$ No

$\Rightarrow$ En caso afirmativo, señalar cuáles:

b. $\square$ El enfermo solicita expresamente no recibir visitas.

c. $\square$ Hay visitas que incomodan o generan malestar emocional al enfermo.

d. $\square$ El enfermo utiliza el móvil u otros dispositivos electrónicos para pasar el tiempo y/o comunicarse con los demás.

e. $\square$ El enfermo demanda compañía y/o atención (p. ej. quejas constantes).

f. Valora la intensidad global de Soledad en el enfermo en una escala (0-10):

$\begin{array}{ccccccccccc}0 & 1 & 2 & 3 & 4 & 5 & 6 & 7 & 8 & 9 & \begin{array}{c}10 \\ \text { NADA }\end{array} \\ \text { MÁXIMA }\end{array}$

CRITERIOS PROVISIONALES DE CORRECCIÓN - EDSOL:

SOLEDAD = ITEM $1+$ ÍTEM $2+$ ÍTEM $3 \Rightarrow[\quad]=(\quad)+(\quad)+(\quad)$

\section{OBSERVACIONES}

- Puntuación máxima: 15.

- Los ítems 1b, 2b i 3b no se suman, pero complementan la información sobre la soledad del enfermo, la posible causa de la misma, y la necesidad o no de derivación a especialistas. El ítem 4 nos da información por parte del profesional sanitario.

Figura 1. Escala de Detección de la Soledad Existencial (EDSOL) 
La escala EDSOL otorga una puntuación total máxima de 15, con un rango que oscila de 0 a 15 formada por la suma de las puntuaciones de las respuestas a los ítems 1, 2 y 3 referentes a la valoración de la percepción de soledad. Las preguntas sobre el por qué de las respuestas del enfermo así como los signos externos de soledad permiten a los profesionales sanitarios conocer mejor las causas posibles de la soledad existencial para llevar a cabo una atención más específica ${ }^{(29,30)}$.

En la elaboración de las preguntas de la escala EDSOL se ha elegido un lenguaje claro y fácilmente comprensible en su formulación. Se ha tenido muy presente la elaboración de preguntas tanto que no generasen o añadiesen más sufrimiento al enfermo ni que las mismas influyan en su respuesta.

Comunicar experiencias de soledad en ocasiones resulta difícil cuando se asocia a un signo de fracaso, o genera vergüenza, así que puede evocar miedo a la muerte ${ }^{(19)}$. Es por esto que en ocasiones, frente a estas experiencias de sufrimiento se organizan mecanismos defensivos para facilitar su afrontamiento ${ }^{(20)}$.

Para analizar la idoneidad de las preguntas de la escala EDSOL (validez de contenido), el cuestionario fue analizado por diferentes psicólogos, expertos en cuidados paliativos pertenecientes al grupo de la Sociedad Catalano-Balear de Cuidados Paliativos que no habían participado en su elaboración inicial. Al 100\% de los mismos les parecieron adecuadas las preguntas. Únicamente se hizo un cambio de estilo en el redactado de una pregunta relacionada con la percepción del profesional sanitario sobre los signos externos de soledad existencial. Para analizar la validez de contenido de las preguntas (validez de facie o aparente) se preguntó a 7 pacientes de dos unidades de cuidados paliativos de diferentes hospitales si comprendían las preguntas, si les parecieron importantes para ellos, si les había molestado el hecho de que se les preguntara por la soledad, o por el contrario, si les había ayudado en su situación (ver Tablas 1 y 2).

Tabla 1. Valoración de las preguntas y su comprensión por parte de los enfermos

\begin{tabular}{|l|l|}
\hline ¿Comprende bien esta pregunta, es clara? & $\begin{array}{l}\square \text { Nada clara } \\
\square \text { Poco clara } \\
\square \text { Regular } \\
\square \text { Clara } \\
\square \text { Muy clara } \\
100 \% \text { clara o muy clara }\end{array}$ \\
\hline $\begin{array}{l}\text { ¿Considera importante que hagamos esta } \\
\text { pregunta? }\end{array}$ & $\begin{array}{l}\square \text { Sí } \square \text { No } \\
100 \% \text { Sí }\end{array}$ \\
\hline ¿Eliminaría esta pregunta? & $\begin{array}{l}\square \text { Sí } \square \text { No } \\
\text { Nadie sugirió eliminar las preguntas }\end{array}$ \\
\hline
\end{tabular}




\section{¿Modificaría esta pregunta?}

$\square$ Sí, cómo

$\square$ No

Nadie sugirió modificar las preguntas

Tabla 2. Valoración de la adecuación de las preguntas por parte del enfermo

\begin{tabular}{|c|c|}
\hline $\begin{array}{l}\text { En general, ¿considera que estas preguntas } \\
\text { pueden ayudar a personas que están en una } \\
\text { situación similar a la suya? }\end{array}$ & $\begin{array}{l}\square \text { Nada } \\
\square \text { Poco } \\
\square \text { Ni mucho Ni poco } \\
\square \text { Bastante } \\
\square \text { Mucho } \\
100 \% \text { bastante/ mucho }\end{array}$ \\
\hline $\begin{array}{l}\text { ¿Considera inadecuadas o le han molestado } \\
\text { las preguntas que acabamos de realizar? }\end{array}$ & $\begin{array}{l}\square \text { Nada } \\
\square \text { Poco } \\
\square \text { Ni mucho Ni poco } \\
\square \text { Bastante } \\
\square \text { Mucho } \\
100 \% \text { nada }\end{array}$ \\
\hline
\end{tabular}

Cómo se puede observar en las tablas 1 y 2 , los 7 pacientes consultados refirieron que comprendieron bien las preguntas sobre soledad, las consideraron importantes, y no las eliminarían ni las modificarían (Tabla 1). Por otra parte, consideraron que las preguntas realizadas sobre soledad les podían ayudar en su situación y consideraban que ninguna de ellas ni les había molestado ni las consideraron inadecuadas (Tabla 2).

\section{Discusión}

A partir del inicio del siglo XXI coincidiendo con el aumento de enfermedades crónicas, el incremento de enfermedades oncológicas, el aumento de la esperanza de vida en la población, una población de pacientes mejor informados y con un mejor control de síntomas somáticos, se observa un aumento de intervenciones psicosociales basadas en marcos referenciales humanistas, espirituales y existenciales que recogen las preocupaciones de los enfermos y de sus familias ${ }^{(27)}$. El paradigma asistencial centrado en la biografía del enfermo, enfatiza la atención sobre el sufrimiento espiritual y/o existencial ${ }^{(30,31)}$. La conciencia de hallarse al final 
de la vida, puede evocar reacciones emocionales y cuestionamientos existenciales que lleven al sufrimiento. Factores como el soporte social y familiar, la esperanza, encontrar un sentido en la vida y poder despedirse, contribuyen al bienestar espiritual $^{(8,32)}$.

Morita y otros investigadores realizaron un análisis exploratorio de las experiencias emocionales de enfermos oncológicos japoneses en situación terminal, con el que detectaron la presencia de sentimientos de soledad existencial y su influencia en el malestar emocional. Estos sentimientos están muy presentes en las situaciones de final de vida y transforman el proceso de morir en una experiencia que produce malestar emocional significativo ${ }^{(33)}$.

Para poder aliviar el sufrimiento que padecen los enfermos al final de la vida, evitando que se desborden sus recursos y se desencadenen síntomas refractarios, es necesario detectar precozmente ese malestar y, para ello, se ha de disponer de instrumentos de validez y fiabilidad contrastadas. En nuestro contexto existen escasos instrumentos especialmente diseñados para un uso clínico en enfermos próximos a la muerte que evalúen la Soledad Existencial. En este sentido, evaluar la presencia e intensidad de la experiencia de soledad existencial permite identificar y priorizar la atención psicosocial en enfermos con malestar emocional moderado y/o severo ${ }^{(33,34)}$.

Por otro lado, a pesar de las dificultades que en muchas ocasiones comporta la evaluación dada la extrema fragilidad de los enfermo ${ }^{(35)}$, la experiencia de evaluación en sí misma genera en la mayoría de los casos el establecimiento de una relación terapéutica con el paciente que estimula la creación de un vínculo con capacidad para proveer un soporte relacional ${ }^{(36)}$ tal y como se ha observado en otras escalas que han evaluado el malestar emocional y han seguido una metodología similar a la de la escala EDSOL propuesta en este documento ${ }^{(24-26)}$.

Dada la situación de vulnerabilidad en la que se encuentran los enfermos al final de la vida, para la utilización de instrumentos de evaluación es fundamental tener presente la necesidad de combinar el máximo rigor científico posible pero con el respeto prioritario que se debe a toda persona que se encuentra en esta situación ${ }^{(31)}$. En este sentido, creemos que nuestra escala permitirá evaluar de forma sistemática la soledad existencial, cubriendo la falta de escalas de evaluación de este tipo y permitiendo un abordaje en mayor profundidad ${ }^{(17)}$, favoreciendo una mejor comprensión de la misma y su incidencia en el malestar emocional del enfermo, favoreciendo una intervención más específica sobre el mismo.

Aunque no se dispone todavía de datos sobre las propiedades psicométricas de la escala, su validez de contenido está avalada tanto por la evaluación realizada por jueces expertos como por enfermos, demostrando, de forma preliminar, la idoneidad de la misma.

El sistema de puntuación establecido para la escala necesita del establecimiento del punto de corte que presente mejor relación posible entre sensibilidad y especificidad, que nos permita discernir entre pacientes que presentan soledad existencial y los que no la presenten. Así mismo, es necesario un análisis en profundidad del resto de propiedades psicométricas de la escala.

Para la evaluación de las propiedades psicométricas de la EDSOL, se realizará un estudio transversal multicéntrico en el cual se administrará la EDSOL a pacientes con enfermedad avanzada al final de la vida que estén ingresados en unidades de cuidados paliativos de diferentes hospitales de Cataluña. Actualmente ya se 
dispone de la aprobación de los comités de ética e investigación clínica (CEIC) de 5 hospitales (Corporación Sanitaria Parc Taulí de Sabadell, Hospital Universitario Sant Joan de Reus, la Fundación Hospital Residencia Sant Camil de Sant Pere de Ribes, el Hospital Universitario Vall d'Hebrón de Barcelona y Consorcio Sanitario de Terrassa).

Deseamos destacar que la escala EDSOL es una propuesta inicial de un instrumento de detección para el cribado de la soledad asistencial en el ámbito de los cuidados paliativos y psicooncología, y que la evaluación clínica posterior, deberá ser desempeñada por el profesional especializado al que sea derivado el enfermo. En este sentido, este trabajo presenta algunas limitaciones relacionadas con el hecho de que no se dispone de resultados concluyentes sobre la escala ya que todavía no se han analizado sus propiedades psicométricas. No obstante, creemos que nuestra escala supondrá un paso más pero importante en la mejora de la atención integral de los enfermos atendiendo de forma holística sus necesidades. Deseamos que los profesionales sanitarios que utilicen la escala propuesta nos vayan indicando con sus comentarios y apreciaciones la bondad y validez de la misma, así como su utilidad en la práctica clínica.

\section{Financiación}

Este trabajo se ha realizado, en parte, gracias a la ayuda PSI2013-43555-R del Ministerio de Economía y Competitividad de España.

\section{Conflicto de intereses}

Los autores declaran que no hay ningún conflicto de intereses.

\section{Referencias bibliográficas}

1. Callahan D. Death and the research imperative. N Engl J Med 2000; 324: 654-6. doi:10.1056/NEJM200003023420910

2. Ministerio de Sanidad y Consumo. Guía de criterios de calidad en cuidados paliativos. Ministerio de Sanidad y Consumo. Madrid; 2002.

3. World Health Organization. Palliative Care. Cancer control: Knowledge into action. WHO guide for effective programmes. Ginebra: World Health Organization; 2007.

4. Sanz J, Gomez Batiste X, Gomez.Sancho M et al. Cuidados Paliativos: recomendaciones de la Sociedad Española de Cuidados Paliativos (SECPAL). Madrid: Ministerio de Sanidad y Consumo; 1993.

5. Cassell E J. The nature of suffering and the goals of medicine. N Engl J Med 1982; 306:639-45. doi: 10.1056/NEJM198209163071224

6. Chapman CR, Gavrin J. Suffering and its relationship to pain. J Palliat Care 1993; 9: 5-13.

7. Bayés R, Arranz P, Barbero J, Barreto P. Propuesta de un modelo integral para una intervención terapéutica paliativa. Med Paliat 1996; 3: 114-21.

8. Krikorian A, Limonero JT, Maté J. Suffering and distress at the end-of-life. Psychooncology 2012; 21: 799-808. doi: 10.1002/pon.2087 
9. Krikorian A, Limonero JT, Román JP, Vargas JJ, Palacio C. Predictors of suffering in advanced cancer. Am J Hosp Palliat Care 2013; 31:534-42. doi:10.1177/1049909113494092

10. Chochinov, H, Dying, dignity and new horizons in palliative end of life care. Cancer J Clin 2006; 56: 84-103. doi: 10.3322/canjclin.56.2.84

11. Strang S. Spiritual/existential issues in palliative care (Doctoral Thesis). Göteborg University; 2002.

12. Boston, P Bruce, A Schreiber, R, Existential suffering in the palliative care setting J Pain Symptom Manage 2011; 41:604-18. doi: 10.1016/j.jpainsymman.2010.05.010

13. Weiss, R. Loneliness: the experience of emotional and social isolation. Cambridge MIT Press; 1973.

14. Weiss, R. Reflections on the present state of loneliness research. J Soci Behav Person 1987; 2: 1-16.

15. Weiss RS. Reflections on the present state of loneliness research. In: Hojat, M. and Crandall R, editors. Loneliness: Theory, research, and applications. Sage: Newbury Park; 1989.p.1-16

16. Limonero JT, Maté J, Mateo D, González-Barboteo J, Bayés R, Bernaus M, et al. Desarrollo de la escala DME-C: una escala para la detección del malestar emocional de los cuidadores principales de personas con enfermedad avanzada o al final de la vida. Ansiedad Estrés; 2016; 22:104-9. doi:10.1016/j.anyes.2016.09.001

17. Sand L, Strang P. Existential loneliness in a palliative home setting. J Palliat Med 2006; 9:1376-1387.

18. Ettema EJ, Derksen LD, Leeuwen E Van. Existential loneliness and end-of-life care: A systematic review. Theor Med Bioeth 2010;31:141-69. doi: 10.1007/s11017-010-9141-1.

19. Mayers AM, Svartberg M. Existential loneliness: A review of the concept, its psychological precipitants and psychotherapeutic implications for HIV-infected women. Br J Med Psychol 2001;74(Pt 4):539-53. doi: 10.1348/000711201161082

20. Yalom ID. Existential psychotherapy. Simon and Schuster: New York;1980.

21. Mijuskovic, Ben. Loneliness in philosophy, psychology and literature. V Gorcum Assen;1979.

22. Maté J. Bayés R. Gonzalez- Barboteo J, Muñoz S, Moreno F, Bayés R. ¿A qué se atribuye que los enfermos oncológicos de una unidad de cuidados paliativos mueran en paz? Psicooncología 2008; 5: 303-21

23. Breitbart W, Gibson C, Poppito SR, Berg A. Psychotherapeutic interventions at the end of life: A focus on meaning and spirituality. Can J Psychiatry 2004; 49:366-72.

24. Maté J, Mateo D, Bayés R, Bernaus M, Casas C, González-Barboteo J, et al. Elaboración y propuesta de un instrumento para la detección de malestar emocional en enfermos al final de la vida. Psicooncología 2009; 6:507-18.

25. Krikorian A, Limonero JT, Corey MT. Suffering assessment: a review of available instruments for use in palliative care. J Palliat Med 2013;16:130-42. doi:10.1089/ jpm.2012.0370

26. Sand L. Existential challenges and coping in palliative cancer care. Experiences of patients and family members. (Doctoral Thesis). Karolinska Institutet: Sweden; 2008.

27. Bayés, R, Limonero JT. Prioridad en el tratamiento de los síntomas que padecen los enfermos oncológicos en situación terminal. Med Paliat 1999; 6: 19-21.

28. Bayés, R. Principios de la investigación psicosocial en cuidados paliativos. En: Die Trill M, López Imedio E, editores. Aspectos psicológicos en cuidados paliativos. La comunicación con el enfermo y la familia. Madrid: Ades, 2000. p. 651-668.

29. Mount BM, Boston P, Cohen R. Healing connections: on moving from suffering to a sense of wellbeing. J Pain Symptom Manage 2007; 33: 372-88. doi: 10.1016/j. jpainsymman.2006.09.014388. 
30. Limonero JT, Mateo D, Maté-Méndez J, González-Barboteo J, Bayés R, Bernaus M, et al. Evaluación de las propiedades psicométricas del cuestionario de Detección de Malestar Emocional (DME) en pacientes oncológicos Gac Sanit 2012; 26:145-52. doi: 10.1016/j. gaceta.2011.07.016

31. Limonero JT, Gil-Moncayo F. Importance of psychological research in palliative care : barriers in its development. Palliat Med Hospice Care Open J 2014; 1:1-3. doi: PMHCOJ/ PMHCOJ-1-101

32. Rokach A, Raan M, Artem S. Bercovith M. The loneliness experience of the dying and those who care for them. Palliat Support Care 2007; 5:153-9. doi: 10.1017/ S1478951507070228

33. Morita T, Tsunoda J, Inoue S, Chihara S. An exploratory factor analysis of existential suffering in Japanese terminally ill cancer patients. Psychooncology 2009; 9:164-8. doi: 10.1002/(SICI)1099-1611(200003/04)9:2<164::AID-PON447>3.0.CO;2-S 